\title{
Dynamics of tree layer composition, tree age and large diameter trees in Habitats Directive Annex I forest habitats in Estonia on the basis of monitoring data collected from 2010-2012
}

\author{
Anneli Palo' ${ }^{*}$ and Mark Gimbutas ${ }^{2}$
}

Palo, A., Gimbutas, M. 2013. Dynamics of tree layer composition, tree age and large diameter trees in Habitats Directive Annex I forest habitats in Estonia on the basis of monitoring data collected from 2010-2012. - Forestry Studies | Metsanduslikud Uurimused 58, 57-73. ISSN 1406-9954.

\begin{abstract}
The protection of late-succession forest habitats and continuous woodlands is the primary goal in Estonian forest nature conservation. Therefore ecological qualities of almost all forest stands in Estonian protected areas are described. Forest patches will be mapped and protected as Annex I forest habitats if they have enough structural and functional qualities. The member states of the European Union report in every six years about the conservation status of European Habitats Directive Annex I habitats. Changes in area as well as changes in habitat type's structure and functions are also declared. In the present paper the dynamics in tree species composition, stand age and larger diameter trees of monitored Annex I forest habitats is analyzed. The mean age of stands is older than the Estonian mean value, but it is only slightly higher than the cutting age of the corresponding tree species. The mean diameter of larger living trees is in general larger than dead wood, which is indicative of the forest succession and historical low-intensive forest management. Currently, conservation activities support natural succession in forests that formerly were managed economically. Therefore, changes in the composition of tree layer species are to be expected. The dominance of spruce and the abundance of broad-leaved trees are increasing in non-managed forest habitats. In contrast, the proportion of pine, birch and aspen decreases significantly. In general the number of tree species in habitat patches will increase; thereby the dominance of certain species will decline. According to the Estonian interpretation of the forest types in the Manual of the Habitats Directive, some stands with changed main tree species will need to be reclassified. Therefore the area of some forest habitat types may increase in the future. On contrary the area and functional quality of some other habitat types may decrease.
\end{abstract}

Key words: Habitats Directive Annex I, forest habitat types, monitoring, succession.

Authors' addresses: ${ }^{1}$ Institute of Ecology and Earth Sciences, University of Tartu, 46 Vanemuise St., Tartu 51014, Estonia; ${ }^{2}$ Institute of Mathematical Statistics, University of Tartu, 2 Juhan Liivi St., Tartu 50409, Estonia; *e-mail: anneli.palo@ut.ee

\section{Introduction}

The protection of late-succession habitats and continuous forest areas (including areas with natural disturbances) is the primary goal in Estonian forest nature conserva- tion (Nature Conservation Act). Longtime human influence on forests and especially rapid intensification of forest clear-cutting and ditching during the last centuries have resulted in low continuity of forest landscapes (Laasimer, 1965; Lõhmus et al., 2004; 
Etverk \& Meikar, 2008; Lõhmus \& Kraut, 2010; Remm et al., 2013). Most especially valuable habitats in Estonia are now maintained within nature conservation areas or special strict protection zones in national parks and landscape protection areas. Felling is prohibited within these areas. Some representative forest habitats are also situated in management zones of protected areas or as key biotopes in State or private forests. Guarantees of future conservation are lower for these habitats because of weaker restrictions on felling (Nature Conservation Act; Kuresoo, 2012).

The tree composition of forest stands in Estonia depends mainly on soil conditions (Laasimer, 1965; Lõhmus, 1984; Paal, 1997, 2002; Kusmin \& Jõgiste, 2006), but also on the stage of development of the given stand and on the degree of disturbance (Lõhmus et al., 2004; Sorohova et al., 2009; Liira et al., 2011) and on recent management/land use techniques before nature conservation measures were established (Etverk \& Meikar, 2008; Liira \& Sepp, 2009; Lõhmus \& Kraut, 2010; Palo et al., 2013). The successional replacement of pioneer trees with other tree species is a natural widespread process in (hemi)boreal forests (Laasimer, 1965; Lõhmus, 1984; Šorohova et al., 2009) and age of these first succession stages is long enough to build habitats with high species diversity and ecological qualities if the forest management intensity remains low (Lõhmus \& Lõhmus, 2008; Liira \& Sepp, 2009; Mežaka et al., 2011; Remm et al., 2013).

In terms of Annex I habitat types, Estonian (hemi)boreal late-successional or virgin forest landscape should be covered with mixed spruce-forests: which corresponds mostly to type Herb-rich spruce forest (9050) with pattern of Western taiga (*9010) and Broad-leaved forests ( $\left.{ }^{*} 9020\right)$. Late-successional peatlands may be also covered with various forest types such as Bog forests ( $\left.{ }^{*} 91 \mathrm{D} 0\right)$, some Western taiga stands or Deciduous swamp forests (*9080). The main forest habitat types can be divided into subtypes on the basis of their landscape features and special forest qualities such as sparse noncultivated Dune forest (2180), fire-influenced species-rich Esker forest (9060), landslips-containing shady and moist Slope forest (*9180) and flooded Alluvial or Riparian forest (*91E0 and 91F0) (Laasimer, 1965; Lõhmus, 1984; Paal, 1997, 2002).

The Estonian forest types of Annex I of the Habitats Directive (92/43/EEC) are defined with reference to forest site types of the Estonian vegetation classification (the classes have relatively uniform soil fertility, acidity and humidity) and cover the range of tree species. In some cases stands from the same Estonian forest site type may belong to different Annex I forest habitat types according to main tree cover (Paal, 2002, 2007). Ecological qualities of almost all forest stands in Estonian conservation areas have been assessed (map scale 1:10 000) to determine whether they are within Habitat Directive Annex I series of forest habitats. Forest patches have been mapped and protected, if they have enough structural and functional qualities such as natural stand composition, presence of old trees, dead wood, indicative species etc. The signs of felling or ditching during last $30-50$ years should also be missing or very infrequent (Paal, 2002; Palo, 2010b).

The member states of the European Union report every six years on the status of habitats and species and state's progress in implementing the goals of the Habitats Directive to the European Commission (European Commission 2006). Initially no country had a monitoring system of forest habitats or even unified understanding of possible indicators (Kuris \& Ruskule, 2006). A redesigned monitoring project of valuable forest habitats in Estonia started at 2006; but the number of plots remained still too low for representative survey (Palo et al., 2005; Palo, 2010a). Since 2010 a new methodology (Liira, 2009) has been used. Principles implemented there can be summarized as follows: random sampling from 
Annex I map database, managed by the Ministry of the Environment; sufficient quantities of plots for data processing for rare habitat types or correspondingly to the area of other habitat types; a list of characteristics which describe the structure of the stand as well as its biological heterogeneity and some special landscape features. Monitoring, defined as identification of changes and interpretation of trends, should show real results after 2018, when data collected on 2010-2012 can be compared those from the period 2013-2018 (Liira, 2010). The understanding of processes which have shaped present forest habitats and will influence future succession, is the key to interpretation of monitored changes in the next periods. So far, only some aspects of development of Annex I forest habitats have been analyzed using actual field work data originating from mapped stands (Palo \& Hoder, 2009; Palo et al., 2010, 2011).

The focus of the first monitoring study and this paper is a description of the present composition and heterogeneity of stands of different forest habitat types as one fundamental assessment criterion of the successful forest conservation is the evaluation of specific structures of the Annex I forest habitats. We analyze stand tree composition, canopy layer trees' age and dynamics of diameter of living and dead wood. Forest management practices which have influenced the development of these stands, as well as future habitat succession and nature conservation details will be discussed. The question posed: is there a contradiction between predetermined habitat classification units and forest succession in the future?

\section{Material and methods}

Habitat types were designated for forest of sampling plots stands as in Paal (2007) and in Palo (2010b); shortened names and codes of habitat types (92/43/EEC Annex I) are given in Figure 1. Sampling was completed according to the method described by Liira (2009): 11 forest habitat types were monitored in 2010-2012; 425 plots with radius $20 \mathrm{~m}$ were described (numbers of study plots per habitat types are presented in Table 1). The full scientific names of the tree species are shown in Table 2 . The plots randomly pre-selected location was only corrected/replaced during fieldworks because of earlier mapping errors or if the plot was unreachable within a reasonable time.

\section{Collected characteristics, used in this paper:}

Tree composition of the canopy layer (the higher, I layer) - expert assessed composition percentile of trees in canopy layer.

Tree composition in the lower sub-canopy layer (II layer) - trees reaching height of $25-75 \%$ of the higher canopy layer, dominant tree species and/or other species list (without high Corylus avellana, with Salix, Sorbus or Prunus padus trees); expert assessment.

Regrowth layer - all trees including seedlings, lower than sub-canopy layer (with Salix, Sorbus and Prunus padus trees, shrub forms are calculated into shrub layer), dominant tree species and/or other species list; expert assessment.

Shrub layer - all shrub species, including high shrubs (e.g. Corylus avellana or Frangula alnus of up to 8-10 m), dominant species and/or other species list; expert assessment.

Mean and maximum age in years of the canopy layer trees - data from forestry database and expert assessment in the field.

Breast height diameter $(\mathrm{cm})$ of three largest living tree trunks and one largest lying dead tree was also measured.

\section{Statistics}

Data analysis was conducted in $\mathrm{R}$ using tests as implemented in package "stats" (R Core Team 2013). Differences in species numbers and trunk diameters were tested by Wilcoxon signed rank test. P-values were adjusted by Holm-Bonferroni method for 
11 habitat types. For each habitat type, tree layer and species the proportion of plots presenting the species in the layer amongst all plots of the habitat type was calculated and is given in Table 1. These figures are frequencies of stands which present the species. Note that missing trees or shrubs in a layer was also treated as a "species" and denoted as " 0 " in Tables 1 and 2 . Differences of proportions between layers were determined by chi-squared test. For each habitat type, p-values were HolmBonferroni corrected and number of tests equals the number of species found in that type, including “ 0 ” (see Table 1 ).

There are three different types of composition formulae in Table 2. For the higher (I) tree layer, coverage proportions of each tree species were estimated in the field, so that mean coverage percentages for habitat types could be calculated. Secondly, plots could be classified by all dominant species and therefore frequencies of dominant species could be calculated. This formula shows species which dominate in each layer in each habitat type. Finally, a composition formula can be obtained by dividing the number of occasions a species was found by the number of all occasions any species was found in that layer. This formula reveals the overall diversity characteristic to habitat types, regardless of species dominance.

\section{Results and discussion}

\section{Stand characteristics in general}

Absence of trees in canopy layer (0-level on Figure 1) is a result of natural disturbances because clear-cutting is prohibited in Annex I habitat areas (Paal, 2007). In other habitat types $0-5 \%$ of plots had a missing higher tree layer (I layer), lower tree (II) layer was missing in $0-37 \%$ of plots, and $0-13 \%$ of plots had no regrowth. (Figure 1, Table 1). The habitat types growing on fertile soils with high probability of natural disturbances (Slope forests, Riparian forests, Wooded pastures) showed varia- ble tree species number (0-10) (Figure 1). According to the canopy layer mean composition formula Broad-leaved forests, Slope forests and Riparian forests with 12 tree species were the richest in species (Table 2). The stand's canopy layer variability and also mean tree species number was lowest on poor soils of Dune and Bog forests, where only 4-5 tree species were present (Figures 1, 2; Table 2). The lower sub-canopy layer mean tree species number was lower (Figure 2) for the Western taiga, Deciduous swamp forests, Alluvial forests and Broad-leaved forests where the regrowth species number was also lower. The regrowth layer tree species number was much higher than species number of sub-canopy layer in peatland forest habitat types (Deciduous swamp forests, Alluvial forests, Bog forests) (Figure 2). Sub-canopy layer, regrowth and/or shrub layer may be absent or species poor if higher tree layers are very dense. Intensive grazing or undergrowth cutting can only in few cases be of major influence in Annex I habitats, as this type of management is rarely permitted. Increasing regrowth and shrub species number comparing with higher tree layer may be result of better light conditions (fast growth after grazing abandonment, natural disturbances or selective cutting) or drainage on peat soils (Lõhmus \& Kraut, 2010; Liira et al., 2011; Palo et al., 2013; Remm et al., 2013).

Comparing the tree species in different layers, in some habitat types a few species may exist in the sub-canopy layer as well as regrowth, but are never found in the canopy layer composition, e.g. tree shaped forms of Sorbus aucuparia and Prunus padus or rarely appearing species like Gray alder. In most habitat types, the sub-canopy layer and regrow th contain spruce and shade-tolerant broad-leaved trees more dominantly and frequently than in their higher canopy layer (Table 2). The shrub layer tends to be more diverse in habitats on dry and moderate rich soils (Herb-rich spruce forests, Wooded pastures, Broad-leaved forests, 
Table 1. Relative frequency of species in layers by habitat type. For each species found at least in one layer of one plot, difference of proportions between tree layers was tested by chi-squared test. For each habitat type, $\mathrm{p}$-values were corrected by Holm-Bonferroni method (***adjusted $\mathrm{p}<$ $\left.0.001,{ }^{* *} 0.001 \leq \mathrm{adj} . \mathrm{p}<0.01,{ }^{*} 0.01 \leq \mathrm{adj} . \mathrm{p}<0.05\right)$. Layers: I - canopy layer, II - sub-canopy layer, $\mathrm{R}$ - regrowth. Tree name abbreviations see in Table 2, habitat type names Figure 1.

Tabel 1. Liikide suhteline sagedus rinnetes elupaigatüüpide kaupa. Iga liigi jaoks, mida leiti vähemalt ühe prooviala ühes rindes, testiti osakaalude erinevust rinnetes hii-ruut testiga. Iga elupaigatüübi kõigi liikide jaoks on $p$-väärtused parandatud Holm-Bonferroni meetodil ( ${ }^{* * *}$ parandatud $p<0.001,{ }^{* *} 0.001$ $\left.\leq p . p<0.01,{ }^{*} 0.01 \leq p . p<0.05\right)$. Rinded: $I$ - esimene rinne, II - teine rinne, $R$-järelkasv. Liiginimede lühendid vt tabel 2, elupaigatüüpide nimed joonis 1 .

\begin{tabular}{|c|c|c|c|c|c|c|c|c|c|c|c|c|c|c|c|c|}
\hline $\begin{array}{l}\text { Habitat } \\
\text { type }\end{array}$ & $\begin{array}{l}\text { No of } \\
\text { plots }\end{array}$ & $\begin{array}{l}\text { Lay- } \\
\text { er }\end{array}$ & $\begin{array}{l}0 " \\
\% \%\end{array}$ & $\begin{array}{c}\text { Pin } \\
\% \\
\end{array}$ & $\begin{array}{c}\text { Pic } \\
\% \\
\end{array}$ & $\begin{array}{c}\text { Bet } \\
\%\end{array}$ & $\begin{array}{c}\text { Pop } \\
\%\end{array}$ & $\begin{array}{c}\text { AlnG } \\
\%\end{array}$ & $\begin{array}{c}\text { Fra } \\
\% \\
\end{array}$ & $\begin{array}{c}\text { Que } \\
\%\end{array}$ & $\begin{array}{c}\text { Ace } \\
\% \\
\end{array}$ & $\begin{array}{l}\text { Til } \\
\% \\
\end{array}$ & $\begin{array}{c}\text { Ulm } \\
\%\end{array}$ & $\begin{array}{c}\text { AlnI } \\
\%\end{array}$ & $\begin{array}{c}\text { Sal } \\
\%\end{array}$ & $\begin{array}{c}\text { SorI } \\
\%\end{array}$ \\
\hline 2180 & 17 & $\begin{array}{l}\mathrm{I} \\
\mathrm{II} \\
\mathrm{R} \\
\end{array}$ & $\begin{array}{c}0 \\
35 \\
12 \\
\end{array}$ & $\begin{array}{l}47^{*} \\
71^{*} \\
\end{array}$ & $\begin{array}{c}6 \\
24 \\
29 \\
\end{array}$ & $\begin{array}{l}12 \\
24 \\
29 \\
\end{array}$ & 0 & 0 & 0 & $\begin{array}{c}0 \\
0 \\
24 \\
\end{array}$ & $\begin{array}{c}0 \\
0 \\
12 \\
\end{array}$ & & & & $\begin{array}{l}0 \\
0 \\
6 \\
\end{array}$ & \\
\hline *9010 & 82 & $\begin{array}{l}\mathrm{I} \\
\mathrm{II} \\
\mathrm{R}\end{array}$ & $\begin{array}{l}3 \\
8 \\
5 \\
\end{array}$ & & $\begin{array}{l}68 \\
85 \\
87\end{array}$ & $37^{* *}$ & $\begin{array}{l}27 \\
10 \\
22 \\
\end{array}$ & $\begin{array}{l}21 \\
19 \\
20\end{array}$ & $\begin{array}{c}1 \\
4 \\
10\end{array}$ & $\begin{array}{c}2^{<0.08} \\
4^{<0.08} \\
14^{<0.08}\end{array}$ & $\begin{array}{c}0^{* * *} \\
6^{* * *} \\
20^{* * *}\end{array}$ & $\begin{array}{c}1 \\
4 \\
11 \\
\end{array}$ & $\begin{array}{l}0 \\
1 \\
2\end{array}$ & $\begin{array}{l}1 \\
1 \\
4\end{array}$ & $\begin{array}{l}0 \\
4 \\
0\end{array}$ & $\begin{array}{l}0 \\
9 \\
1\end{array}$ \\
\hline *9020 & 34 & $\begin{array}{l}\mathrm{I} \\
\mathrm{II} \\
\mathrm{R}\end{array}$ & $\begin{array}{c}0 \\
15 \\
12 \\
\end{array}$ & $\begin{array}{l}24^{*} \\
3^{*} \\
0^{*}\end{array}$ & $\begin{array}{l}44 \\
44 \\
50 \\
\end{array}$ & & $\begin{array}{l}65^{*} \\
26^{*} \\
32^{*}\end{array}$ & $\begin{array}{c}12 \\
9 \\
6 \\
\end{array}$ & $\begin{array}{l}32 \\
26 \\
47 \\
\end{array}$ & $\begin{array}{l}79^{* * *} \\
26^{* * *} \\
24^{* * *}\end{array}$ & $\begin{array}{l}24 \\
35 \\
50 \\
\end{array}$ & $\begin{array}{l}88 \\
41 \\
\end{array}$ & $\begin{array}{l}26 \\
24 \\
\end{array}$ & $\begin{array}{l}6 \\
9 \\
\end{array}$ & 0 & $\begin{array}{l}3 \\
0 \\
0\end{array}$ \\
\hline 9050 & 35 & $\begin{array}{l}\mathrm{I} \\
\mathrm{II} \\
\mathrm{R}\end{array}$ & $\begin{array}{l}0 \\
6 \\
3 \\
\end{array}$ & $\begin{array}{c}34^{* * *} \\
0^{* * *} \\
0^{* * *}\end{array}$ & $\begin{array}{c}100 \\
94 \\
94 \\
\end{array}$ & & $\begin{array}{l}60^{*} \\
20^{*} \\
34^{*}\end{array}$ & 17 & $\begin{array}{c}9 \\
17 \\
26 \\
\end{array}$ & & & $\begin{array}{c}6 \\
20 \\
29 \\
\end{array}$ & $\begin{array}{c}0 \\
11 \\
9 \\
\end{array}$ & $\begin{array}{c}6^{*} \\
26^{*} \\
40^{*} \\
\end{array}$ & 3 & \\
\hline 9060 & 32 & $\begin{array}{l}\text { I } \\
\text { II } \\
\text { R } \\
\end{array}$ & $\begin{array}{l}0 \\
9 \\
9 \\
\end{array}$ & & $\begin{array}{l}84 \\
88 \\
88 \\
\end{array}$ & $\begin{array}{l}59 \\
34 \\
28 \\
\end{array}$ & & 0 & $\begin{array}{c}3 \\
3 \\
16 \\
\end{array}$ & & & $\begin{array}{l}3 \\
3 \\
3 \\
\end{array}$ & 0 & $\begin{array}{c}3 \\
13 \\
16 \\
\end{array}$ & $\begin{array}{c}9 \\
22 \\
6 \\
\end{array}$ & \\
\hline 9070 & 63 & $\begin{array}{l}\mathrm{I} \\
\mathrm{II} \\
\mathrm{R}\end{array}$ & $\begin{array}{c}0^{*} \\
21^{*} \\
13^{*}\end{array}$ & $\begin{array}{l}27^{*} \\
10^{*} \\
8^{*}\end{array}$ & $\begin{array}{l}21 \\
17 \\
27 \\
\end{array}$ & $\begin{array}{l}78^{<0.06} \\
57^{<0.06} \\
51^{<0.06}\end{array}$ & $\begin{array}{l}60 \\
35 \\
40 \\
\end{array}$ & $\begin{array}{l}24 \\
19 \\
25\end{array}$ & $\begin{array}{l}48 \\
41 \\
56\end{array}$ & $\begin{array}{l}46 \\
24 \\
43 \\
\end{array}$ & $\begin{array}{c}3^{*} \\
16^{*} \\
27^{*}\end{array}$ & $\begin{array}{l}2 \\
0 \\
3\end{array}$ & 5 & $\begin{array}{c}5 \\
14 \\
19 \\
\end{array}$ & $\begin{array}{l}0 \\
6 \\
3\end{array}$ & $\begin{array}{l}0 \\
2 \\
2\end{array}$ \\
\hline *9080 & 31 & $\begin{array}{l}\text { I } \\
\text { II } \\
\text { R } \\
\end{array}$ & $\begin{array}{c}0^{* *} \\
32^{* *} \\
6^{* *} \\
\end{array}$ & $\begin{array}{c}45^{* * *} \\
3^{* * *} \\
0^{* * *} \\
\end{array}$ & $\begin{array}{l}48 \\
55 \\
74 \\
\end{array}$ & $\begin{array}{l}94^{* *} \\
42^{* *} \\
58^{* *}\end{array}$ & $\begin{array}{c}29^{<0.08} \\
3^{<.08} \\
10^{<0.08} \\
\end{array}$ & $\begin{array}{l}74 \\
48 \\
74 \\
\end{array}$ & $\begin{array}{l}10^{<0.07} \\
13^{<0.07} \\
39^{<0.07}\end{array}$ & $\begin{array}{c}3 \\
0 \\
13 \\
\end{array}$ & $\begin{array}{c}0 \\
0 \\
10 \\
\end{array}$ & $\begin{array}{l}0 \\
0 \\
6 \\
\end{array}$ & & $\begin{array}{l}3 \\
3 \\
0 \\
\end{array}$ & $\begin{array}{l}0 \\
0 \\
3 \\
\end{array}$ & \\
\hline *9180 & 25 & $\begin{array}{l}\text { I } \\
\text { II } \\
\text { R }\end{array}$ & $\begin{array}{l}4 \\
0 \\
0 \\
\end{array}$ & $\begin{array}{c}20 \\
0 \\
4 \\
\end{array}$ & $\begin{array}{l}36 \\
28 \\
40 \\
\end{array}$ & $\begin{array}{l}44 \\
20 \\
12 \\
\end{array}$ & $\begin{array}{c}32 \\
12 \\
8 \\
\end{array}$ & 24 & $\begin{array}{l}28 \\
12 \\
28 \\
\end{array}$ & $\begin{array}{c}12 \\
8 \\
4 \\
\end{array}$ & 56 & $\begin{array}{l}48 \\
36 \\
28 \\
\end{array}$ & $\begin{array}{l}60 \\
48\end{array}$ & $\begin{array}{l}28 \\
40 \\
44 \\
\end{array}$ & $\begin{array}{c}24 \\
16 \\
0 \\
\end{array}$ & \\
\hline *91D0 & 29 & $\begin{array}{l}\text { I } \\
\text { II } \\
\text { R } \\
\end{array}$ & $\begin{array}{c}3^{*} \\
37^{*} \\
11^{*}\end{array}$ & $\begin{array}{l}97^{* * *} \\
44^{* * *} \\
67^{* * *} \\
\end{array}$ & $\begin{array}{l}24 \\
26 \\
55 \\
\end{array}$ & $\begin{array}{l}45 \\
44 \\
74 \\
\end{array}$ & $\begin{array}{l}0 \\
0 \\
4 \\
\end{array}$ & $\begin{array}{l}7 \\
4 \\
4 \\
\end{array}$ & & & & & & & $\begin{array}{l}0 \\
4 \\
0 \\
\end{array}$ & \\
\hline *91E0 & 42 & $\begin{array}{l}\mathrm{I} \\
\mathrm{II} \\
\mathrm{R}\end{array}$ & $\begin{array}{c}5 \\
22 \\
5\end{array}$ & $\begin{array}{l}5 \\
0 \\
0\end{array}$ & $\begin{array}{l}19^{* *} \\
53^{* *} \\
60^{* *}\end{array}$ & $\begin{array}{l}86^{* * *} \\
35^{* * *} \\
28^{* * *}\end{array}$ & $\begin{array}{c}17^{<0.08} \\
0^{<0.08} \\
5^{<0.08}\end{array}$ & $\begin{array}{l}90 \\
70 \\
85\end{array}$ & $\begin{array}{c}7^{* *} \\
23^{* *} \\
48^{* *}\end{array}$ & $\begin{array}{l}2 \\
0 \\
3\end{array}$ & $\begin{array}{l}0 \\
0 \\
8\end{array}$ & $\begin{array}{c}0^{<0.08} \\
5^{<0.08} \\
18^{<0.08}\end{array}$ & $\begin{array}{c}0 \\
2 \\
15 \\
\end{array}$ & $\begin{array}{ll}2 & 1 \\
0 & \\
5 & \end{array}$ & $\begin{array}{c}17^{<0.08} \\
0^{<0.08} \\
5^{<0.08}\end{array}$ & \\
\hline 91F0 & 37 & $\begin{array}{l}\text { I } \\
\text { II } \\
\text { R }\end{array}$ & $\begin{array}{c}3 \\
11 \\
5\end{array}$ & $\begin{array}{l}8 \\
0 \\
0\end{array}$ & $\begin{array}{l}24 \\
53 \\
44 \\
\end{array}$ & $\begin{array}{c}70^{* * *} \\
39^{* * *} \\
8^{* * *}\end{array}$ & $\begin{array}{c}49^{* *} \\
6^{* *} \\
19^{* *}\end{array}$ & $\begin{array}{l}54 \\
55 \\
55\end{array}$ & $\begin{array}{l}35 \\
36 \\
58\end{array}$ & $\begin{array}{c}16 \\
8 \\
11 \\
\end{array}$ & $\begin{array}{c}5 \\
14 \\
22 \\
\end{array}$ & $\begin{array}{l}11 \\
17 \\
14\end{array}$ & $\begin{array}{l}14 \\
33 \\
43 \\
\end{array}$ & $\begin{array}{l}32 \\
36 \\
57\end{array}$ & $\begin{array}{c}11 \\
8 \\
8 \\
\end{array}$ & \\
\hline
\end{tabular}

Esker forests), where about 5 species grow on each plot and more than 20 different species can be found in each habitat type. Poorest in species are Bog forests where the shrub layer was absent in $55 \%$ of plots and the mean number of species was one (Figures 1, 2; Table 2).
Monitored Annex I forest habitat types had typical or moderately diverse tree and shrub layer composition compared to Estonian forest site type mean values (Adermann, 2012: Table 33.1), as far as they are comparable with each other (Paal, 1997, 2002, 2007; Kusmin \& Jõgiste, 2006). 
Table 2. Composition formulas of habitat types (\%): 0 - trees/shrubs missing or no dominants; Layers: I - canopy layer, II - sub-canopy layer, R - regrowth, S - shrubs. Habitat type names see Figure 1. Ace - Acer platanoides, AlnG - Alnus glutinosa, AlnI - Alnus incana, Bet - Betula pendula and $B$. pubescens, Cor - Corylus avellana, Dap - Daphne mezereum, Fra - Fraxinus excelsior, Frn - Frangula alnus, Jun - Juniperus communis, Lon - Lonicera xylosteum, Pic - Picea abies, Pin - Pinus sylvestris, Pop - Populus tremula, Que - Quercus robur, Rha - Rhamnus cathartica, RibA - Ribes alpinum, Rib - Ribes sp., Rosa - Rosa sp., Rub - Rubus idaeus, Sal - Salix sp., SorA - Sorbus aucuparia, SorI - Sorbus intermedia, SorR - Sorbus rupicola, Til - Tilia cordata, Ulm - Ulmus glabra and U. laevis, Vib - Viburnum opulus.

Tabel 2. Elupaigatüüpide koosseisuvalemid (\%): 0 - puud või põõsad puuduvad või pole võimalik määrata domineerivat liiki; Rinded: I - I puurinne, II - II puurinne, $R$ - järelkasv, S - põõsarinne. Elupaigatüüpide nimed vt joonis 1 .

\begin{tabular}{|c|c|c|c|c|}
\hline $\begin{array}{l}\text { Habitat } \\
\text { type }\end{array}$ & $\begin{array}{l}\text { Mean composition } \\
\text { formula in I layer } \\
(\%) \text { and total sp. } \\
\text { No in (...) }\end{array}$ & Layer & $\begin{array}{l}\text { Composition formula calcu- } \\
\text { lated by dominant species } \\
\text { frequencies }(\%)\end{array}$ & $\begin{array}{l}\text { Composition formula calculated by all men- } \\
\text { tioned species frequencies }(\%)\end{array}$ \\
\hline \multirow[t]{4}{*}{2180} & \multirow{4}{*}{$\begin{array}{l}\text { 96Pin } 2 \text { AlnG 2Bet } \\
+ \text { Pic,Pop } \\
\text { (5) }\end{array}$} & $\mathrm{I}$ & 94Pin $6 A \ln G$ & 77Pin 9Bet 5AlnG 5Pop 4Pic \\
\hline & & II & 35-0 41Pin 12Pic 6Bet 6AlnG & 25-0 33Pin 17Pic 17Bet 4Alng 4Fra \\
\hline & & $\mathrm{R}$ & 18-0 47Pin 29Pic 6Ace & 6-0 37Pin 15Pic 15Bet 12Que 6Ace 3AlnG \\
\hline & & $\mathrm{S}$ & 18-0 47 Jun 29SorA 6Frn & $\begin{array}{l}\text { 10-0 31Jun 19SorA 16Frn 6Lon 6RibA 3Cor } \\
\text { 3Pru 3Rib 3Vib }\end{array}$ \\
\hline \multirow[t]{5}{*}{ *9010 } & \multirow{5}{*}{$\begin{array}{l}\text { 47Pin 20Bet 19Pic } \\
\text { 10Pop 3AlnG } \\
\text { +Que,Til,Fra,AlnI } \\
\text { (9) }\end{array}$} & & \multirow{5}{*}{$\begin{array}{l}\text { 3-0 43Pin 22Pic 19Bet 10Pc } \\
1 \text { AlnG } \\
\text { 8-0 82Pic 5Bet 3Pin 1AlnG } \\
1 \text { Ace } \\
\text { 8-0 67Pic 8Bet 8Pin 6Ace } \\
\text { 1AlnG 1Til 1Fra } \\
\text { 19-0 33SorA 20Cor 9Jun } \\
\text { 5Pru 3Lon 3Betula nana } \\
\text { 1Rub 1Sal }\end{array}$} & Pin 26Pic 24Bet 10Pop 8AlnG \\
\hline & & II & & 3-0 40Pic 20 Pin 9Alng 7 Pin 5Pop 4SorA \\
\hline & & $\mathrm{R}$ & & 2-0 35Pic 14Bet 8Pop 8AlnG 8Ace 6Pin \\
\hline & & & & 6Que 5Til 4Fra 2AlnI 1Ulm 1SorA \\
\hline & & $\mathrm{S}$ & & $\begin{array}{l}\text { 7-0 24SorA 14Frn 13Cor 8Pru 8Lon 5Rub } \\
\text { 5Jun 4Vib 3RibA 3Dap 2Rha 1Betula nana } \\
\text { 1Sal 1Rib+Rosa,SorI, Berberis vulgaris }\end{array}$ \\
\hline \multirow[t]{4}{*}{ *9020 } & \multirow{4}{*}{$\begin{array}{l}\text { 37Que 15Pop } \\
\text { 12Bet 8Fra 8Til } \\
\text { 6Pic 5Ace 3AlnG } \\
\text { 3Ulm 1AlnI 1Pin } \\
\text { 1SorI } \\
(12)\end{array}$} & $\mathrm{I}$ & $\begin{array}{l}\text { 59Que 11Pop 9Bet 9Til 6Fra } \\
\text { 3Ace 3AlnG }\end{array}$ & $\begin{array}{l}\text { 20Que 16Pop 16Bet 11Pic 9Til 8Fra 6Pin } \\
\text { 6Ace 4Ulm 3AlnG 1AlnI+SorI }\end{array}$ \\
\hline & & II & $\begin{array}{l}\text { 17-0 26Pic 15Til 12Ace 9Fra } \\
\text { 6Ulm 3AlnG 3AlnI 3SorA } \\
\text { 3Que 3Mal }\end{array}$ & $\begin{array}{l}\text { 5-0 14Pic 12Til 11Ace 9Bet 9Pop 9Fra 8Ulm } \\
\text { 8Que 5SorA 3AlnG 2AlnI 2Mal 1Pin 1Sal } \\
\text { 1Pru }\end{array}$ \\
\hline & & $\mathrm{R}$ & & $\begin{array}{l}\text { 3-0 16Ace 16Pic 15Fra 13Til 10Pop 7Ulm } \\
\text { 7Que 4Bet 3AlnI 3Pru 2AlnG 1SorA }\end{array}$ \\
\hline & & S & 85 Cor 12 Pru 3SorA & $\begin{array}{l}\text { 23Cor } 16 \text { SorA } 14 \text { Pru } 13 \text { Lon } 8 \text { Vib } 7 \text { RibA } \\
\text { 5Frn 4Rub 2Dap 2Cornus sanguinea 1Rosa } \\
\text { 1Rha } 1 \text { Rubus caesius 1Berberis vulgaris } \\
\text { 1Crataegus sp. }\end{array}$ \\
\hline \multirow[t]{4}{*}{9050} & \multirow{4}{*}{$\begin{array}{l}\text { 62Pic 16Bet 10Pop } \\
\text { 5Pin 3AlnG 1Que } \\
\text { 1AlnI 1Til 1Fra } \\
\text { +Ace,Sal } \\
\text { (11) }\end{array}$} & & 88Pic 6Bet 6Pop & $\begin{array}{l}\text { 30Pic 25Bet 18Pop 10Pin 5Alng 3Que 3Fra } \\
\text { 2AlnI 2Til 1Ace 1Sal }\end{array}$ \\
\hline & & II & $\begin{array}{l}\text { 8-0 74Pic 9AlnI 3Pop 3Til } \\
\text { 3Ace }\end{array}$ & \\
\hline & & $\mathrm{R}$ & $\begin{array}{l}\text { 3-0 60Pic 11Til 11Ace 9AlnI } \\
\text { 6SorA }\end{array}$ & $\begin{array}{l}\text { 1-0 26Pic 12Ace 11AlnI 9Pop 8Til 7Fra } \\
\text { 7Que 7SorA 5Bet 4AlnG 2Ulm 1Pru }\end{array}$ \\
\hline & & S & 63Cor 17SorA 14Pru 6Frn & $\begin{array}{l}\text { 19SorA 17Cor 13Lon 12Pru 9RibA 8Rub } \\
\text { 7Dap 6Frn 3Vib 2Rha 1Jun 1Sal 1Rib } \\
\text { 1Sambucus racemosa 1Amelanchier sp. }\end{array}$ \\
\hline \multirow[t]{4}{*}{9060} & \multirow{4}{*}{$\begin{array}{l}\text { 41Pin 33Pic 16Bet } \\
\text { 6Pop 3Que 1Sal } \\
\text { +Fra,Ace,Til,AlnI } \\
\text { (10) }\end{array}$} & I & & $\begin{array}{l}\text { 29Pic 26Pin 20Bet 13Pop 5Que 3Sal 1Fra } \\
\text { 1Ace 1Til 1AlnI }\end{array}$ \\
\hline & & II & $\begin{array}{l}\text { 10-0 66Pic 6Ace 3Bet 3AlnI } \\
\text { 3Pin 3SorA 3Que 3Pru }\end{array}$ & $\begin{array}{l}\text { 3-0 29Pic 11Bet 11SorA 10Que 7Ace 7Sal } \\
\text { 6Pop 4Pin 4AlnI 3Pru 2Ulm 1AlnG 1Fra 1Til }\end{array}$ \\
\hline & & $\mathrm{R}$ & $\begin{array}{l}\text { 10-0 69Pic 3Ace 3Pin 3SorA } \\
\text { 3Que 3Pop 3AlnI 3Fra }\end{array}$ & $\begin{array}{l}\text { 3-0 29Pic 16Ace 9Bet 8Pop 8Que 7SorA } \\
\text { 5Fra 5AlnI 3Ulm 2Sal 2Pru 1Til 1Pin }\end{array}$ \\
\hline & & $S$ & 75Cor 16SorA 6Jun 3Pru & $\begin{array}{l}\text { 21SorA 20Cor 15Lon 13RibA 7Rub 7Pru } \\
\text { 5Dap 4Jun 3Frn 2Rib 1Vib 1Sal 1SorR }\end{array}$ \\
\hline
\end{tabular}




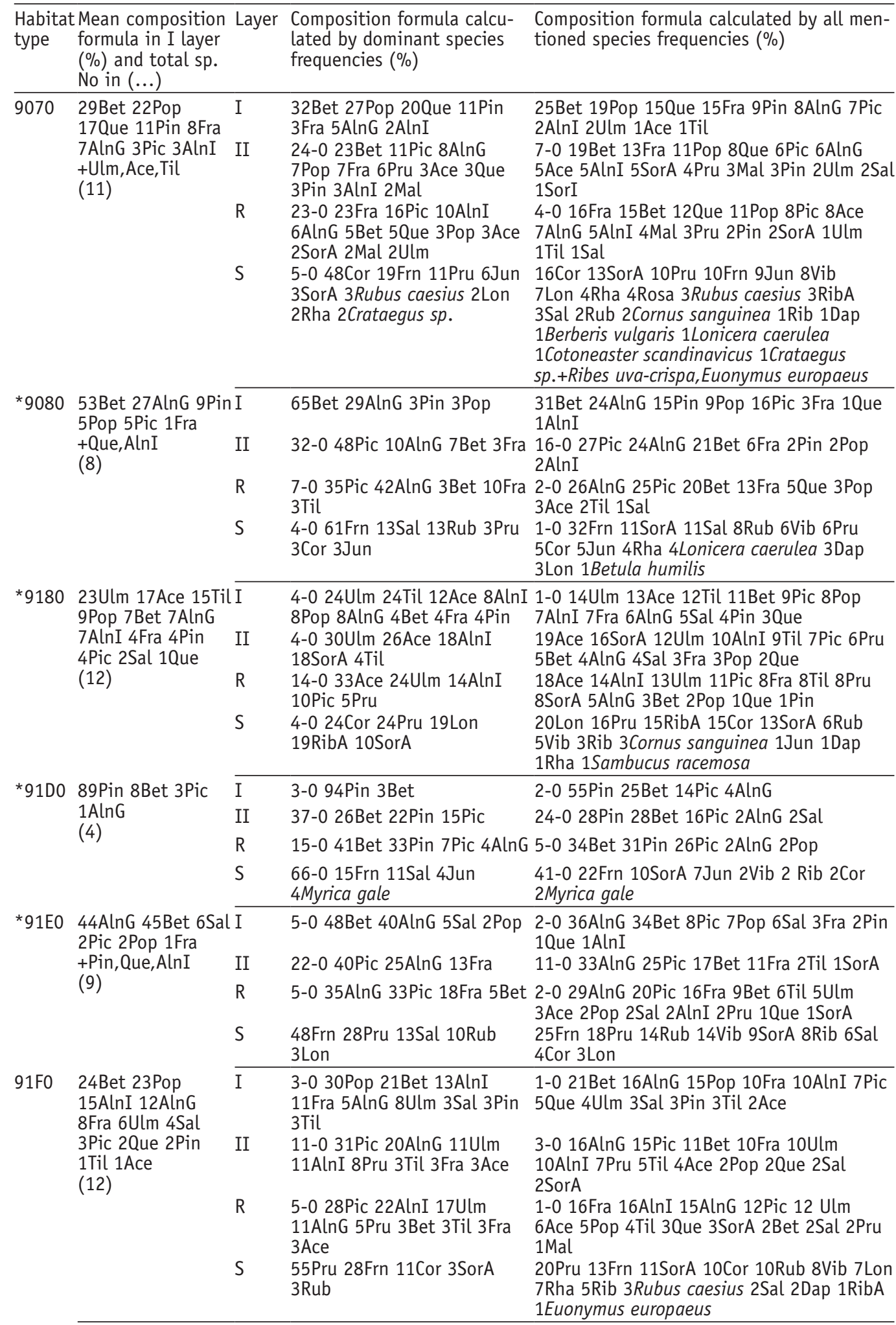




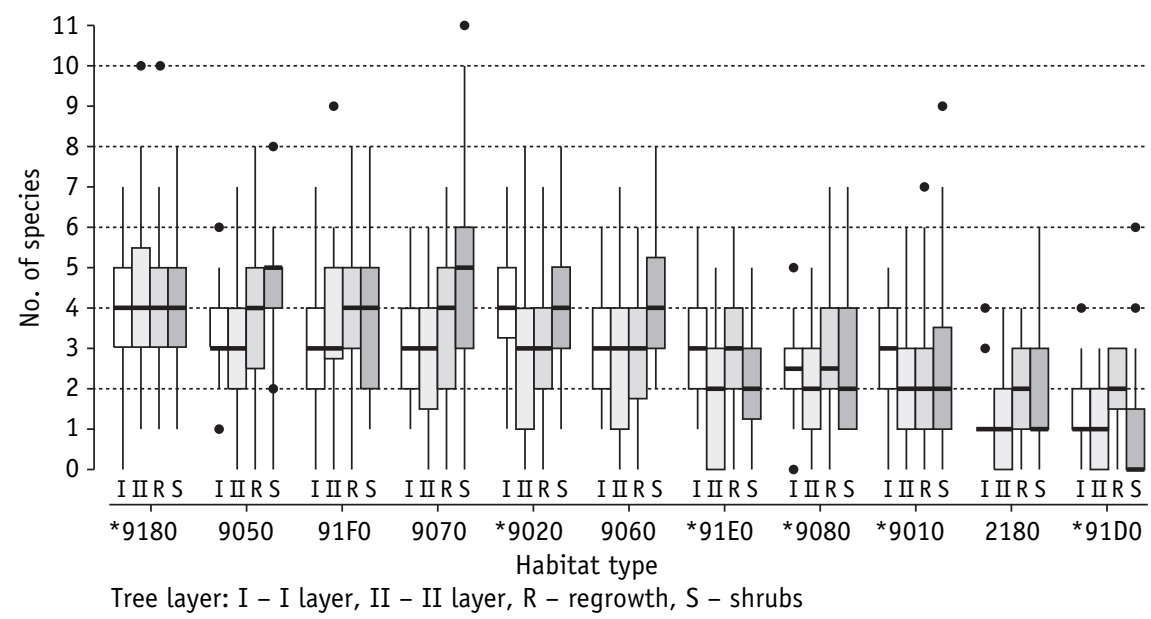

Figure 1. Number of species by layer and habitat type. Bold line in the box represents median, upper and lower edge of the box represent quartiles, whiskers or dots show extreme values. Sample sizes in habitat types ( $\mathrm{n}$ ) are given in Table 1. Annex I habitat type codes and short names: 2180 - Dune forests; *9010 - Western taiga; 9050 - Herb-rich spruce forests; 9060 - Esker forests; 9070 - Wooded pastures; *9080 - Deciduous swamp forests; *9180 - Slope forests; *91D0 - Bog forests; *91E0 - Alluvial forests; 91F0 - Riparian forests.

Joonis 1. Liikide arv rinnete ja elupaigatüüpide kaupa. Jäme joon kastis näitab mediaani, kasti ülemine ja alumine serv näitavad kvartiile, vurrud või punktid näitavad vähimaid ja suurimaid väärtuseid. Valimi suurus elupaigatüüpides (n) on antud tabelis 1. Loodusdirektiivi elupaikade koodid ja lühinimed: 2180 - Luitemetsad; *9010 - Vanad loodusmetsad; 9050 - Rohundirikkad kuusikud; 9060 - Oosimetsad; 9070 - Puiskarjamaad; *9080 - Soo-lehtmetsad; *9180 - Pangametsad; *91D0-Rabametsad; *91E0 - Lammi-lodumetsad; $91 F 0$ - Uhtvallimetsad.

The differences in number of average species between tree layers (canopy and subcanopy layer and regrowth; Figures 1, 2) are not pronounced for most habitat types and the tree species number in boreal or hemiboreal forests remains limited (Šorohova et al., 2009).

The mean age of trees in habitats (Table 3) is higher than in Estonian forests in general (for example pine is 72 years and spruce 58 years as described by Adermann, 2012: Table 12) and the permitted cuttingage for different tree species is reached (RTL 2007, 2, 16). In protected sites the age should be higher than the Estonian mean, but directly comparable forestry data are not available (Mets 2011). The age of the trees is an important but not the only indi- cator of a high degree of representativity (Palo, 2010b; Palo et al., 2011), since the protection of Annex I forest habitats has more objectives than only preserving old forests (92/43/EEC). Modern forest protection approaches combine species conservation with preservation of natural processes and substrate dynamics (Lõhmus et al., 2004, Brūmelis et al., 2011; Winter, 2012; Götmark, 2013). The age of the trees stays temporarily low also in stands which are regenerating after natural disturbances, but there are always sufficient microhabitats for specialized species (Šorohova et al., 2009; Lõhmus \& Kraut, 2010). Nevertheless, low tree age in Annex I forest habitats is mostly associated with too few substrates or too little volume of them (Palo et al., 2010). 


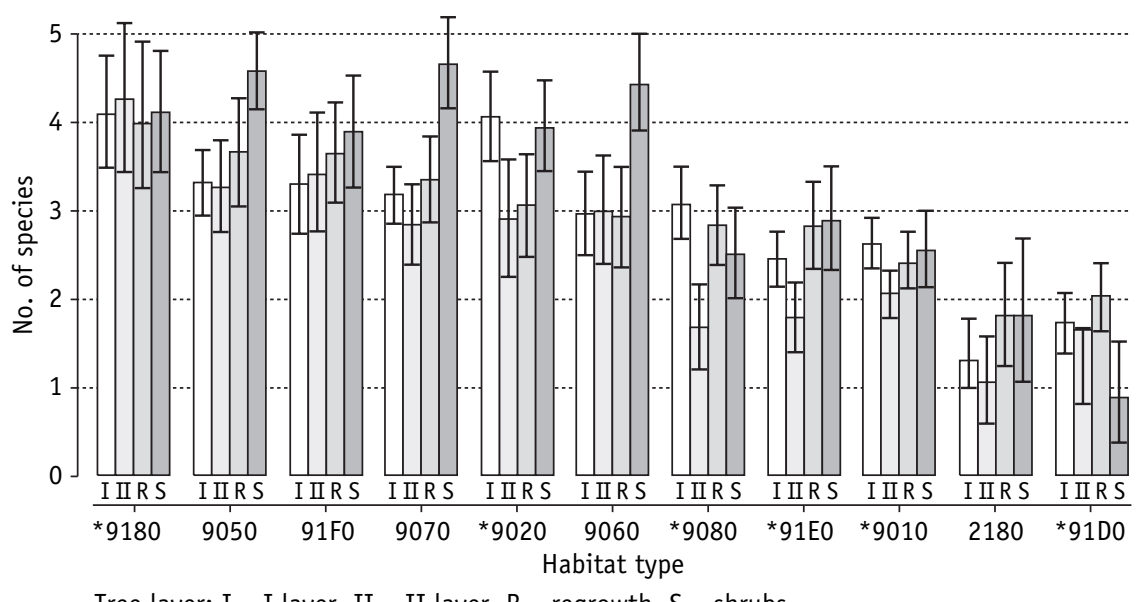

Tree layer: I - I layer, II - II layer, R - regrowth, S - shrubs

Figure 2. Mean number of species by layer and habitat type. Error bars indicate the $95 \%$ percentile bootstrap confidence interval of the mean (10,000 bootstrap samples). Layers: I - canopy layer, II - sub-canopy layer, $\mathrm{R}$ - regrowth, $\mathrm{S}$ - shrubs. Sample sizes of habitat types ( $\mathrm{n}$ ) are given in Table 1 and names of habitat types by Figure 1.

Wilcoxon signed rank test statistics and Holm-Bonferroni adjusted $\mathrm{p}$-values: comparing subcanopy layer and canopy layer: ${ }^{*} 9010-p=0.00013 ;{ }^{*} 9080-p=0.00081 ;{ }^{*} 91 \mathrm{E} 0-p=0.024$; ${ }^{*} 9020-p=0.050$; comparing sub-canopy layer and regrowth: ${ }^{*} 9080-p=0.0090 ;{ }^{*} 91 \mathrm{E} 0-p=$ $0.00087 ;{ }^{*} 91 \mathrm{D} 0-\mathrm{p}=0.021$; comparing regrowth and canopy layer: ${ }^{*} 9020-\mathrm{p}=0.039$.

Joonis 2. Keskmine liikide arv rinnete ja elupaigatüüpide kaupa. Vearibad näitavad keskmise $95 \%$ bootstrapusaldusintervalle (10 000 bootstrap-valimi keskmise 0,025-kvantiil ja 0,975-kvantiil). Rinded: I - esimene rinne, II - teine rinne, $R$ - järelkasv, $S$ - alusmets. Valimi suurus elupaigatüüpides ( $n$ ) on antud tabelis 1 ja elupaigatüüpide nimetused joonisel 1.

Wilcoxoni astakmärgitest Holm-Bonferroni parandusega parandatud $p$-väärtused: II rinde võrdlus I-ga: ${ }^{*} 9010-p=0.00013 ;{ }^{*} 9080-p=0.00081 ;{ }^{*} 91 E 0-p=0.024 ;{ }^{*} 9020-p=0.050$; II rinde võrdlus järelkasvuga: *9080 $-p=0.0090 ;{ }^{*} 91 E 0-p=0.00087 ;{ }^{*} 91 D 0-p=0.021$; I rinde võrdlus järelkasvuga: ${ }^{*} 9020-p=0.039$.

Uniformly low mean ages of birch (7074 years) were present in Slope forests, Alluvial swamp forests and Wooded pastures or oak (103-115 years) in Wooded pastures, Herb-rich spruce forests, Esker forests and Slope forests. Low spruce, birch and aspen mean ages (50-75 years) in Wood pastures are probably a sign of the overgrowing which started in many areas after the Second World War. It was expected to find very old pines in Dune forests, or old pines, spruce, birch, aspen and black alder in Western taiga and old oaks in Broadleaved forests. Slope forests and Broad- leaved forests containing old pine trees are however rare, but were nevertheless represented in the sample (150-180 years) (Table 3). The latter forests are probably overgrown Wooded pastures, as some of them also contain old pines with a maximum age of 140 years. In future, the mean age of trees in unmanaged forest habitats may reach the level of the mean biological age of the trees as shown in Table 3.

The mean diameter of the three largest living trunks was in most habitat types significantly higher than the diameter of the largest dead wood (Wilcoxon signed 
Table 3. Mean age (years) and maximal age of I, canopy layer trees in habitat types by species. Only plots presenting a species are included in calculations; confidence interval is given if there were at least three such plots. +/- - half-width of 95\% confidence interval of the mean. Maximal ages at least 100 are indicated. Tree name abbreviations see in Table 2, habitat type names see Figure 1.

Tabel 3. Esimese rinde puude keskmine ja maksimaalne vanus aastates liikide ja elupaigatüüpide kaupa. Arvestatud on neid proovialasid, kus vastavat liiki puid leidus; usaldusintervall on näidatud siis, kui selliseid alasid oli vähemalt kolm. +/- - aritmeetilise keskmise $95 \%$ usaldusintervalli poollaius. Maksimaalne vanus on antud, kui ületas 100 aastat. Liiginimede lühendeid vt tabel 2, elupaigatüüpide nimed joonis 1.

\begin{tabular}{|c|c|c|c|c|c|c|c|c|c|c|c|c|c|c|c|c|c|}
\hline H. type & \multicolumn{2}{|l|}{2180} & \multicolumn{3}{|c|}{ *9010 } & \multicolumn{3}{|c|}{ *9020 } & \multicolumn{3}{|c|}{9050} & \multicolumn{3}{|c|}{9060} & \multicolumn{3}{|c|}{9070} \\
\hline $\begin{array}{l}\text { Tree } \\
\text { sp. }\end{array}$ & $\begin{array}{l}\text { Mean +/- } \\
\text { age }\end{array}$ & $\begin{array}{l}\text { Max } \\
\text { age }\end{array}$ & $\begin{array}{c}\text { Mean } \\
\text { age }\end{array}$ & $+/-$ & $\begin{array}{l}\text { Max } \\
\text { age }\end{array}$ & $\begin{array}{c}\text { Mean } \\
\text { age }\end{array}$ & $+/-$ & $\begin{array}{l}\text { Max } \\
\text { age }\end{array}$ & $\begin{array}{c}\text { Mean } \\
\text { age }\end{array}$ & $n+/-$ & $\begin{array}{l}\text { Max } \\
\text { age }\end{array}$ & $\begin{array}{c}\text { Mean } \\
\text { age }\end{array}$ & $+/-$ & $\begin{array}{l}\text { Max } \\
\text { age }\end{array}$ & $\begin{array}{c}\text { Mean } \\
\text { age }\end{array}$ & $+/-$ & $\begin{array}{l}\text { Max } \\
\text { age }\end{array}$ \\
\hline $\begin{array}{l}\text { Pin } \\
\text { Pic } \\
\text { Bet } \\
\text { Pop } \\
\text { AlnG } \\
\text { Fra } \\
\text { Que } \\
\text { Ace } \\
\text { Til } \\
\text { Ulm } \\
\text { AlnI } \\
\text { Sal } \\
\end{array}$ & \begin{tabular}{|ll}
117.1 & 27.4 \\
60 & \\
65 & \\
60 & \\
70 &
\end{tabular} & 250 & \begin{tabular}{|c}
104.8 \\
93.2 \\
93 \\
81.4 \\
85.6 \\
70 \\
80 \\
\\
80 \\
80
\end{tabular} & $\begin{array}{c}6.6 \\
5.9 \\
5.4 \\
5.4 \\
10.5 \\
\\
25\end{array}$ & $\begin{array}{l}200 \\
160 \\
160 \\
110 \\
140\end{array}$ & \begin{tabular}{|c}
115 \\
98 \\
92.6 \\
76.8 \\
82.5 \\
68.2 \\
133.6 \\
72.5 \\
89.1 \\
96 \\
30
\end{tabular} & $\begin{array}{c}22.3 \\
13.9 \\
11.3 \\
8.9 \\
50.9 \\
10.3 \\
16.6 \\
11.6 \\
15.7 \\
28.5\end{array}$ & $\begin{array}{l}150 \\
140 \\
140 \\
130 \\
130 \\
100 \\
300 \\
100 \\
130 \\
130\end{array}$ & \begin{tabular}{|c}
93.3 \\
97.4 \\
97.2 \\
81.4 \\
98.3 \\
86.7 \\
112.5 \\
90 \\
90 \\
\\
50 \\
70 \\
\end{tabular} & $\begin{array}{ll}3 & 15.4 \\
4 & 7.5 \\
2 & 7.9 \\
4 & 7.9 \\
3 & 20.3 \\
7 & 37.9 \\
5 & 32.8\end{array}$ & $\begin{array}{l}140 \\
150 \\
140 \\
120 \\
120 \\
100 \\
130\end{array}$ & \begin{tabular}{|c}
114.4 \\
96.3 \\
91.6 \\
71.7 \\
\\
80 \\
106 \\
50 \\
70 \\
70 \\
53.3 \\
\end{tabular} & $\begin{array}{r}20.8 \\
\\
37.9 \\
\end{array}$ & $\begin{array}{l}200 \\
180 \\
110 \\
120\end{array}$ & \begin{tabular}{|c}
70.6 \\
63.8 \\
69.8 \\
53.8 \\
72 \\
56.3 \\
115.2 \\
75 \\
80 \\
66.7 \\
30
\end{tabular} & $\begin{array}{c}11.7 \\
12.7 \\
5.0 \\
5.7 \\
13.6 \\
7.2 \\
9.9 \\
\\
\\
62.5 \\
24.8\end{array}$ & $\begin{array}{l}140 \\
100 \\
100 \\
100 \\
100 \\
100 \\
150\end{array}$ \\
\hline $\begin{array}{l}\text { H. type } \\
\text { Tree } \\
\text { sp. }\end{array}$ & $\begin{array}{l}{ }^{*} 9080 \\
\text { Mean } \\
\text { age }\end{array}$ & $\begin{array}{l}\text { Max } \\
\text { age }\end{array}$ & $\begin{array}{c}\text { Mean } \\
\text { age }\end{array}$ & $\begin{array}{l}\text { *9180 } \\
+/-\end{array}$ & $\begin{array}{l}\text { Max } \\
\text { age }\end{array}$ & $\begin{array}{c}\text { Mean } \\
\text { age }\end{array}$ & $\begin{array}{c}\text { *91D0 } \\
+/-\end{array}$ & $\begin{array}{l}\text { Max } \\
\text { age }\end{array}$ & $\begin{array}{c}\text { Mean } \\
\text { age }\end{array}$ & $\begin{array}{l}* 91 \mathrm{EO} \\
n+/-\end{array}$ & $\begin{array}{l}\text { Max } \\
\text { age }\end{array}$ & $\begin{array}{c}\text { Mean } \\
\text { age }\end{array}$ & $\begin{array}{l}91 F 0 \\
+/-\end{array}$ & $\begin{array}{c}\text { Max } \\
\text { age }\end{array}$ & & & \\
\hline $\begin{array}{l}\text { Pin } \\
\text { Pic } \\
\text { Bet } \\
\text { Pop } \\
\text { AlnG } \\
\text { Fra } \\
\text { Que } \\
\text { Ace }\end{array}$ & $\begin{array}{|cc|}84.3 & 17.6 \\
78.7 & 14.3 \\
80.3 & 6.6 \\
54.4 & 11.6 \\
73.5 & 7.0 \\
60 & 43.0 \\
100 & \\
\end{array}$ & $\begin{array}{l}160 \\
160 \\
130 \\
\\
130\end{array}$ & $\begin{array}{c}124 \\
76.7 \\
76 \\
78.8 \\
68.3 \\
76.7 \\
103.3 \\
78.5 \\
91.8 \\
70 \\
52.9 \\
46.7\end{array}$ & $\begin{array}{c}45.2 \\
12.7 \\
14.4 \\
12.2 \\
15.4 \\
21.7 \\
62.5 \\
13.0 \\
9.4 \\
14.2 \\
11.6 \\
14.3\end{array}$ & $\begin{array}{l}100 \\
130 \\
120 \\
120 \\
100\end{array}$ & $\begin{array}{c}101.4 \\
80 \\
80 \\
\\
90\end{array}$ & $\begin{array}{l}10.8 \\
28.3 \\
15.4\end{array}$ & $\begin{array}{l}170 \\
130 \\
120\end{array}$ & \begin{tabular}{|c}
80 \\
67.5 \\
74.2 \\
70 \\
71.8 \\
55 \\
110
\end{tabular} & $\begin{array}{cc}5 & 11.6 \\
2 & 4.7 \\
23.0 \\
3.6 \\
27.6\end{array}$ & $\begin{array}{l}100 \\
100 \\
100\end{array}$ & $\begin{array}{c}53.3 \\
72.2 \\
75.4 \\
75 \\
70.5 \\
75.4 \\
135 \\
85 \\
77.5 \\
108 \\
53.3 \\
82.5\end{array}$ & $\begin{array}{c}57.4 \\
17.5 \\
6.8 \\
9.2 \\
8.7 \\
10.6 \\
42.4 \\
\\
52.6 \\
32.1 \\
10.3 \\
41.8\end{array}$ & $\begin{array}{l}120 \\
110 \\
200 \\
100 \\
120 \\
140\end{array}$ & & & \\
\hline
\end{tabular}

rank test, Holm-Bonferroni-corrected pvalues $>0.05$ only for Dune forests and Slope forests) (Figure 3). In the stand level the diameter gap between three largest living trunks and diameter of lying dead wood (Figure 3) is also characteristic for initial forest succession phases (self-thinning), but in some paludifying forests the volume (diameter) of new tree generation also remains lower (Nilsson et al., 2002; Simard et al., 2007; Šorohova et al., 2009; Lõhmus \&
Kraut, 2010). In addition, this gap may be a sign of earlier low-intensive forest management effect in the forests which are now protected (Tyrrell \& Crow, 1994). In strictly protected areas, the mean value of the diameter gap as well as the mean age of trees in every habitat type should balance in time, provided that the frequency of natural disturbances and paludification processes does not change rapidly. 


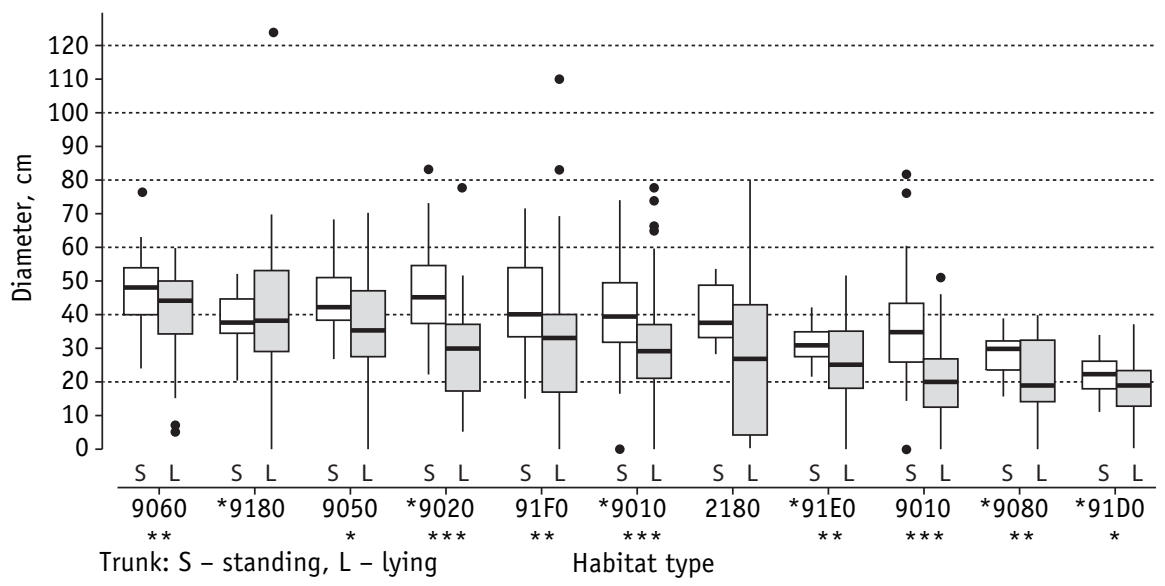

Figure 3. Mean diameter of three thickest trunks and maximal diameter of lying dead wood by habitat type. Graph elements as in Figure 1. Star denotes habitat types for which corresponding diameters are significantly different (Wilcoxon signed rank test with Holm-Bonferroni correction: ***adjusted $p<0.001,{ }^{* *} 0.001 \leq$ adj. $p<0.01,{ }^{*} 0.01 \leq$ adj. $\left.p<0.05\right)$. Sample sizes of habitat types $(n)$ are given in Table 1 and names of habitat types by Figure 1 .

Joonis 3. Kolme jämedaima puu keskmine ja suurim lamapuidu läbimõõt elupaigatüüpide kaupa. Graafikuelementide seletus nagu joonisel 2. Tähega on märgitud need elupaigatüübid, mille puhul vastavad läbimõõdud on oluliselt erinevad (Wilcoxoni astakmärgitest Holm-Bonferroni parandusega: *** parandatud $p<0.001,{ }^{* *} 0.001 \leq p . p<0.01,{ }^{*} 0.01 \leq p . p<0.05$ ). Valimi suurus elupaigatüüpides $(n)$ on antud tabelis 1 ja elupaigatüüpide nimetused joonisel 1.

\section{Future changes of tree}

\section{composition in habitat types}

Data from Tables 1 and 2 shows the current tree layer composition of each habitat type. As most of Annex I habitats are nowadays protected and therefore unmanaged, natural processes are taking place. The aging trees on canopy layer will be replaced by trees from lower layers and hence one can predict changes in species composition of plots which belong in that habitat type now.

Pine dominates in the canopy layer of Dune forests. Spruce is common in the sub-canopy tree layer and regrowth, but like deciduous species rare in the canopy layer. A comparatively species-rich shrub layer with presence of oak, ash and maple indicate as one possible successional direction Esker forests. The common succession is towards the typical Western taiga with pine, spruce and some birches in higher tree layer and Juniperus communis and Sorbus aucuparia in the shrub layer. Groundwater influenced areas always have Gray alder (Laasimer, 1965; Paal, 2007) in their stand composition (Tables 1, 2).

Western taiga habitats at present include more pine stands than are likely in the future, when spruce will become more dominant and frequent. Aging and low frequency of birch trees in sub-canopy layer will, in the absence of disturbance, decrease the presence of birches in the stands for many years; whereas the frequency of broad-leaved trees will rise, especially maple and oak (Tables 1,2). Currently there is an insignificant trend of increasing elm and lime frequency as showed also Lõhmus \& Kraut (2010).

In Broad-leaved forests $s_{L}$ the dominance of oak, aspen and birch clearly shows a 
decreasing trend. In the next generation, spruce is therefore likely become more frequent. The dominance of shade-tolerant broad-leaved species will also increase. Light-demanding pine will totally disappear from the tree composition, as it is a relict tree species from overgrown Wooded pastures 9070. Corylus avellana dominated in shrub layer and it's frequency on plots was very high, it may even prevent the survival of shade-tolerant regrowth for a while (Tables 1, 2).

Herb-rich Spruce forests will lose pine trees from their composition and frequency of birch and aspen will decrease. The dominance of spruce will also decrease, but it will stay as a frequent tree species. The stands will be mixed spruce-forests with many deciduous tree species in all layers and a species-rich shrub layer, where Corylus avellana is frequent and mostly dominant (Tables 1, 2).

The untouched Esker forests will develop into mixed spruce forests. The frequency of pine will substantially decrease, yet it will still remain in the composition. Together with shade-tolerant broadleaved trees and species-rich shrub layer, light-dependent oak, birch and aspen will remain quite frequent (Tables 1, 2).

Management of Wooded pastures is required, but if this ceases, they will develop into diverse, mixed forest with dominance of birch, spruce, ash and Black alder (Table 2). Frequency of pine and birch will decrease, whereas maple will increase (Table 1).

Pine will disappear from the stand composition of Deciduous swamp forests and birch will lose its dominance. Apart from a consistent presence of Black alder and a higher percentage of spruce, the diversity and percentage of broad-leaved trees will increase (Tables 1, 2). Some changes are perhaps due to drainage (Remm et al., 2013).

Slope forests were the only habitat type with a stable tree composition, as the difference between the species composition of the layers was low. In future, spruce will be somewhat more frequent whereas birch, aspen and pine will become less common (Tables 1, 2).

The Bog forest habitat type includes raised bog pine forests and coniferousdominated mixed transitional bog forests. Theoretically, raised bog pine forests develop towards wooded bog or the succession may be disrupted by fires or changing water regimes. In all cases, the dominant tree species in the canopy layer, as well as in regrowth, will be pine. Transitional bog forests have variable stand composition and their succession is related to the surrounding mire-landscape evolution. There are three possible scenarios: quick acidification by Sphagnum-peat accumulation and formation of bog pine forests or continual transitional bog forests with quite stable mixed tree composition structure. Alternatively an inflow of nutrient rich water leads to the formation of deciduous swamp forests with a deep peat layer (Laasimer 1965). Because of these naturally opposite processes, the mean tree layer composition of this habitat type, even in strictly protected forests, is unpredictable. It is possible that spruce and birch may become more abundant in future because of ongoing drainage effects (Tables 1, 2) (Remm et al., 2013).

The main factors shaping the composition of Alluvial swamp forests are probably the impact of historical drainage, cutting and traditional agricultural land use on wooded mires (Laasimer, 1965; Etverk \& Meikar, 2008; Remm et al., 2013). Therefore, the dominance and frequency of birch, aspen and willow decreases and that of spruce, lime and ash increases (Tables 1, 2).

The young age of trees and lack of continual stands can be detected in habitat type Riparian forests. Theoretically, their higher tree layer composition should show a variety of broad-leaved trees (Laasimer, 1965; Paal, 1997, 2007), but at present first successional phases with aspen and birch are dominant. Their dominance and frequency will greatly decrease in following generations, at the same time the percent- 
age of spruce will increase (Table 2). The lack of large dead wood and the lower age of trees are also common for places with historical, traditional land use (Tables 1, 2,3).

The canopy layer tree composition is expected to change in future in many habitat types as many stands are not yet in a mature phase. Even potentially late-successional habitat types such as Western taiga and Herb-rich spruce forests contained many pioneer-trees in stands tree composition, low age and a low quantity of dead wood. Most forests have probably only been for the last 20-50 years without any felling or extensive grazing. In hemiboreal forests successional changes lead in many cases to changes in the main tree species and together with natural paludification causing shifts to other Annex I forest habitat types (Figure 4). This may be the reason why Latvia has not accepted the Herb-rich spruce forests habitat type but has designated spruce forests as Western taiga (Kuris \& Ruskule, 2006).

Empirical predictions for the possible succession of habitat types, as shown in Figure 4, are of course theoretical. The changes in individual patches may vary in

Figure 4. Theoretical ways of succession of Annex I forest habitats, based on knowledge about the development of forest site types and communities (Laasimer, 1965; Lõhmus, 1984; Paal, 1997, 2002; Kusmin \& Jõgiste, 2006) and mapping knowhow (Palo, 2010b). Continuous arrow - common natural succession; dashed arrow - rarely occurring succession or changes after natural disturbances (fire, windthrow, flooding). Habitat type names see Figure 1.

Joonis 4. Metsa elupaigatüüpide loodusliku suktsessiooni suunad, mis põhinevad metsakoosluste ja kasvukohatüüpide uurimustele (Laasimer, 1965; Lõhmus, 1984; Paal, 1997, 2002; Kusmin \& Jõgiste, 2006) ning elupaikade kaardistamise kogemustele (Palo, 2010b). Pideva joonega nool - tavaline looduslik suktsessioon; katkendjoonega nool - harvaesinev suktsessioon või muutus pärast looduslikke häiringuid (tuli, tormiheide, üleujutus). Elupaigatüüpide nimed vt joonis 1. a large scale through natural disturbances (Šorohova et al., 2009). However, in this paper we have considered the frequency of disturbances to be relatively stable. The majority of general effects described above is well known and understood, such as dead wood dynamics or the differences between pioneer-trees, spruce and the dominance of broad-leaved trees in old-growth compared with managed stands (Kohv \& Liira, 2005; Liira \& Sepp, 2009; Lõhmus \& Kraut, 2010; Liira et al., 2011; Palo et al., 2013). Some of the possible changes suggested above need further study: (1) there is no information why some present natural Dune forests and other dry pine stands are rapidly overgrowing with spruce or deciduous trees and shrubs, especially maple, oak, Sorbus aucuparia and Corylus avellana; (2) in regrowth of forests from different habitat types more oaks are now present than formerly (Liira et al., 2011); (3) there is also a discussion on how long a dense Corylus avellana layer can persist in some habitat types and how this will influence oldgrowth structure in the future, as trees do not regenerate under the dense shrub layer; (4) further study is also needed to determine whether a decline in abundance of

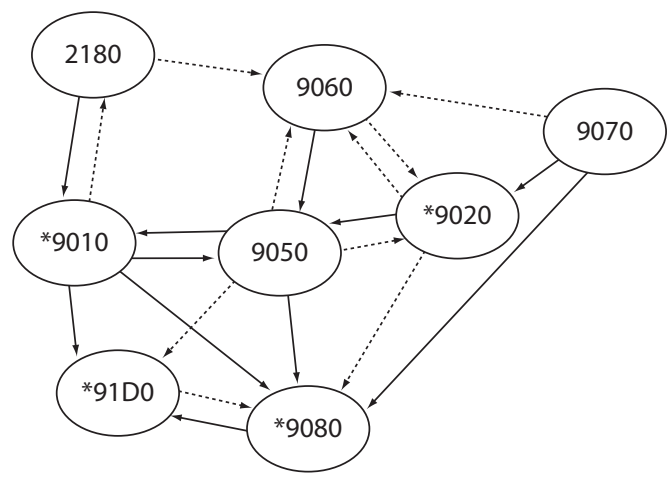


aspen and ash will lead to a decrease in biodiversity of lichens, bryophytes, saproxylic fungi or beetles (Jüriado et al., 2003; Mežaka et al., 2011; Queloz et al., 2011; Lõhmus \& Runnel, 2014).

Taking into account that the main objective of forest nature conservation in Estonia is to protect natural processes and latesuccessional or typical hemi-boreal species (Nature Conservation Act; Lõhmus et al., 2004), the progressive natural changes in forest habitats is not a primary concern in nature conservation policy. Nevertheless, it might become a policy issue that the relative representation of some habitat types is changing. By contrast, functionality, structural complexity of stands and connectivity (European Commission 2006; Kuris \& Ruskule, 2006) between forest habitats in general is improving. This situation has occurred because most of the valuable large forest areas are already protected and there are few possibilities for the establishment of new forest conservation areas. In addition, private land owners are resisting the establishment of new reserves, and felling on State land has been too intensive. It is unlikely that the pressure of construction projects, agriculture, or political factors will affect the nature conservation status of present forest habitats on existing strictly protected land. However, intensive cutting of surrounding forests and increasing recreational activities on protected areas are realistic threats for the functionality of some forests. However, the ecological value of many forests outside protected areas are endangered because of the factors discussed above (Sustainability 2010; Kuresoo, 2012).

\section{Conclusions}

The results from the monitoring project have indicated the long-term human influences on the current structure and composition of forest habitats in Estonia. The mean age of trees in most habitat types was generally comparable with the clear-cut- ting age of managed forests and only a few stands contained trees with age approaching their biological limits. The large diameter gap in most habitat types between the largest living trunks of the trees and the size of fallen dead wood, relates to the degree of maturity of the stands and the extent of selective forest cutting before the establishment of nature conservation sites. Slope forests were in the best natural condition and the stand dynamics within them were relatively well balanced. Young and first succession phase stands were prevalent in Riparian forests.

Strongly human-influenced forest habitat types such as Dune forests (fire, grazing, removal of individual trees, recreation), Esker forests (fire, grazing, selective cutting) and Wooded pastures (grazing, selective cutting) urgently need management plans to ensure the maintenance of the influence of traditional practices on the structure and composition of the stands. The results of the monitoring exercise show there are currently too many stands with ongoing natural succession.

The tree species composition of different layers can be used to infer potential future changes in forest habitat types that would develop with continuous natural succession (Western taiga, Broad-leaved forests, Herbrich spruce forests, Deciduous swamp forests, Bog forests). A decrease of pine-, birchand aspen-dominated stands is expected, as well as a potential increase in dominance or frequency of spruce and broad-leaved trees. Very dense stands of shrubs (especially Corylus avellana) are likely to dominate for some time on rich soils.

The predicted successional changes in tree composition of the forests will demand a reclassification of some patches in terms of the list Annex I habitats. In order to further understand the influence of natural processes and both present and former management practices on ecological quality and functionality of forest habitats, it is suggested that data should be compared from strictly protected sites with no felling 
allowed, with other forests designated as Annex I habitat where some management is allowed.

Acknowledgments. This paper was supported by Target Funding project No. IUT2-16 of the Ministry of Education and Science of Estonia; field work and initial data analysis were financed by Estonian Environmental Board and Ministry of Environment. We are grateful to anonymous reviewer for very useful comments on the manuscript; and Dagmar Hoder, Merit Otsus and Robert Gerald Henry Bunce for editing the text.

\section{References}

92/43/EEC. - Council Directive 92/43/EEC of 21 May 1992 on the conservation of natural habitats and of wild fauna and flora. [WWW document]. - URL http:/ / eur-lex.europa.eu/LexUriServ/LexUriServ.do?uri=CELEX:31992L0043:EN:HTML. [Accessed May 10, 2010].

Adermann, V. (ed.) 2012. Eesti metsad 2010. Metsavarude hinnang statistilisel valikmeetodil. (The Estimation of Forest Sources by Statistical Sampling Methodology). Keskkonnateabe Keskus. Tallinn, 157 pp. [WWW document]. - URL http://www. keskkonnainfo.ee/failid/Eesti_metsad_2010.pdf. [Accessed September 23, 2013]. (In Estonian).

Brūmelis, G., Jonsson, B.G., Kouki, J., Kuuluvainen, T., Šorohova, E. 2011. Forest naturalness in northern Europe: perspectives on processes, structures and species diversity. - Silva Fennica, 45(5), 807-821.

Etverk, I., Meikar, T. 2008. Era- ja põllumajandusmetsad Eestis. (Private and plantation forests). SA Erametsakeskus, 248 pp. (In Estonian).

European Commission 2006. Assessment, Monitoring and Reporting under Article 17 of the Habitats Directive. Explanatory Notes \& Guidelines. EU, Brussels. [WWW document]. - URL http:/ / www. artdata.slu.se/filer/gybs/notes_guidelines_report_art17_final.pdf [Accessed May 10, 2010].

Götmark, F. 2013. Habitat management alternatives for conservation forests in the temperate zone: Review, synthesis, and implications. - Forest Ecology and Management, 306, 292-307.

Jüriado, I., Paal, J., Liira, J. 2003. Epiphytic and epixylic lichen species diversity in Estonian natural forests. - Biodiversity and Conservation, 12, 1587-1607.

Kohv, K., Liira, J. 2005. Anthropogenic effects on vegetation structure of the boreal forest in Estonia. - Scandinavian Journal of Forest Research, 20, 122-134.
Kuresoo, L. (ed.) 2012. Ülevaade Eesti metsandusest 2009-2011. Estonian Fund for Nature (ELF). [WWW document]. - URL http://issuu.com/ ivar $/$ docs $/$ metsaraamat $/ 1$ ? $=0$. [Accessed August 10, 2013]. (In Estonian).

Kuris, M., Ruskule, A. 2006. Favorable conservation status of boreal forests: monitoring, assessment, management. Baltic Environmental Forum. Tallinn. 39 pp.

Kusmin, J., Jõgiste, K. 2006. Eesti metsakasvukohatüüpide floristilise sarnasuse hindamise erinevatest meetoditest. (Different methods of the estimation of floristic similarity in Estonian forest site types). - Forestry Studies/Metsanduslikud Uurimused 44, 52-59. (In Estonian).

Laasimer, L. 1965. Eesti NSV taimkate. (Vegetation of the Estonian SSR). Tallinn, 397 pp. (In Estonian).

Liira, J. 2009. Olemasolevate koosluste seiremetoodikate hindamine ning soovitusi Natura 2000 elupaikade seisundi seiremetoodika. Tartu Ülikooli Kirjastus. [WWW document]. - URL http:/ / www. ut.ee/lkb/avalikud_failid/Liira_2009.pdf._[Accessed May 10, 2010]. (In Estonian).

Liira, J. 2010. Metsaelupaikade seire kameraalsete metoodikate arendus. (Practical analysis of data obtained from the monitoring of forest habitats). Tartu Ülikool, 25 pp. (In Estonian).

Liira, J., Sepp, T. 2009. Indicators of structural and habitat natural quality in boreo-nemoral forests along the management gradient. - Annales Botanici Fennici, 46(4), 308-325.

Liira, J., Sepp, T., Kohv, K. 2011. The ecology of tree regeneration in mature and old forests: combined knowledge for sustainable forest management. - Journal of Forest Research, 16(3), 184-193.

Lõhmus, A., Kohv, K., Palo, A. and Viilma, K. 2004. Loss of old-growth, and the minimum need for strictly protected forests in Estonia. - Ecological Bulletins, 51, 401-411.

Lõhmus, A., Kraut, A. 2010. Stand structure of hemiboreal old-growth forests: characteristic features, variation among site types, and a comparison with FSC-certified mature stands in Estonia. - Forest Ecology and Management, 260(1), 155-166.

Lõhmus, A., Lõhmus, P. 2008. First-generation forests are not necessarily worse than long-term managed forests for lichens and bryophytes. - Restoration Ecology, 16(2), 231-239.

Lõhmus, A., Runnel, K. 2014. Ash dieback can rapidly eradicate isolated epiphyte populations in production forests: a case study. - Biological Conservation, 169, 185-188.

Lõhmus, E. 1984. Eesti metsakasvukohatüübid. (Estonian forest site types). Eesti NSV Agrotööstuskoondise Info- ja Juurutusvalitsus. Tallinn, $121 \mathrm{pp}$. (In Estonian).

Mežaka, A., Brūmelis, G., Piterāns, A. 2012. Tree and stand-scale factors affecting richness and composition of epiphytic bryophytes and lichens in 
deciduous woodland key habitats. - Biodiversity and Conservation, 22(12), 3221-3241.

Mets 2011. 2013. Aastaraamat. (Yearbook). Keskkonnateabe Keskus. Tartu, 230 pp. [WWW document]. - URL http:/ / www.keskkonnainfo.ee/failid/aastaraamat_METS_2011.pdf. [Accessed September 23, 2013]. (In Estonian).

Nature Conservation Act. RT I 2004, 38, 258. [WWW document]. - URL https://www.riigiteataja. ee/en/eli/ee/Riigikogu/act/508112013010/consolide. [Accessed May 10, 2013].

Nilsson, S.G., Niklasson, M., Hedin, J., Aronsson, G., Gutowski, J.M., Linder, P., Ljungberg, H., Mikusiński, G., Ranius, T. 2002. Densities of large living and dead trees in old-growth temperate and boreal forests. - Forest Ecology and Management, 161, 189-204.

Paal, J. 1997. Eesti taimkatte kasvukohatüüpide klassifikatsioon. (Classification of Estonian vegetation site types). Tallinn, 297 pp. (In Estonian).

Paal, J. 2002. Estonian forest site types in terms of the Habitat Directive. - Baltic Forestry, 8, 21-27.

Paal, J. 2007. Loodusdirektiivi elupaigatüüpide käsiraamat. (Handbook of the Habitat Directive habitats). Tallinn, 308 pp. (In Estonian).

Palo, A. 2010a. Ohustatud taimekoosluste (Natura 2000 kooslused) seire: metsaelupaigad. (Endangered plant communities (communities of Natura 2000) monitoring). - Liiv, K., Antso, K. (eds.). Eesti keskkonnaseire 2009. Keskkonnateabe Keskus, Tallinn, p. 78. (In Estonian).

Palo, A. 2010b. Loodusdirektiivi metsaelupaikade inventeerimise juhend. (Habitats Directive forest habitat inventory guide). - Viilma, K., Paal, J., Türnpu, T., Maamets, L., Otsus, M., Animägi, A. (eds.). Käsikiri. Tellija: Eesti Keskkonnaministeerium. (Manuscript. Subscriber: Estonian Ministry of the Environment). [WWW document]. - URL http://www.envir.ee/1168606. [Accessed April 10, 2011]. (In Estonian).

Palo, A., Abner, O., Kalamees, R., Ploompuu, T., Roosaluste, E., Truus, L., Vellak, K. 2005. NATURA 2000 elupaikade seire integreerimine looduse mitmekesisuse seire taimekoosluste seire allprogrammi. (Natura 2000 habitat monitoring, the integration of biodiversity monitoring to the vegetation monitoring sub-program). - Frey, T. (ed.). Eesti XV Ökoloogiapäev "Natura 2000 Eestis", 22. aprill 2005, Tartu (Estonian XV Ecology Day "Natura 2000 in Estonia", Apr. 22, 2005, Tartu), 25-35. (In Estonian).

Palo, A., Hoder, D. 2009. Millal saaks Alutagusest taas põlismets ehk kas ajaloolistest kaartidest on abi Loodusdirektiivi metsaelupaikade piiritlemisel. (When will Alutaguse be old-growth forest again... are historical maps usable for localizing FloraFauna-Habitat directive forest habitats?). - Mander, Ü., Uuemaa, E., Pae, T (eds.). Publicationes Instituti Geographici Universitatis Tartuensis
(Uurimusi eestikeelse geograafia 90. aastapäeval), 108, 330-343. (In Estonian).

Palo, A., Hoder, D., Liira. J. 2011. Re-evaluation of stand indicators for the assessment of the representativity status of the Natura 2000 habitat type forests. - Estonian Journal of Ecology, 60(3), 209-224.

Palo, A., Ivask, M., Liira, J. 2013. Biodiversity composition reflects the history of ancient semi-natural woodland and forest habitats - Compilation of an indicator complex for restoration practice. - Ecological Indicators, 34, 336-344.

Palo, A., Liira, J., Hoder, D. 2010. Kvaliteediastmete eristamisest loodusdirektiivi metsaelupaigatüüpides. (Quality assessment of forest habitat types (Annex I priority habitat types of the Council Directive 92/43/EEC)). - XI Eesti Ökoloogiakonverents „Elurikkus ja ökosüsteemid” 8.-9. aprill 2010, Tartu. (Estonian XI Ecology Conference "Biodiversity and Ecosystems", Apr. 8.-9, 2010, Tartu). [WWW document]. - URL http://www. lote.ut.ee/geo/okoloogiakonverents. [Accessed April 20, 2013]. (In Estonian).

Queloz, V, Grünig, C.R., Berndt, R., Kowalski, T., Sieber, T.N., Holdenrieder, O. 2011. Cryptic speciation in Hymenoscyphus albidus. - Forest Pathology, 41(2), 133-142.

R Core Team 2013. R: A language and environment for statistical computing. R Foundation for Statistical Computing, Vienna, Austria. [WWW document]. - URL http://www.R-project.org/. [Accessed December 24, 2013].

Remm, L., Lõhmus, P., Leis, M., Lõhmus, A. 2013. Long-term impacts of forest ditching on nonaquatic biodiversity: conservation perspectives for a novel ecosystem. - PLoS ONE, 8(4), e63086.

RTL 2007, 2, 16. Rules of Forest Management. [WWW document]. - URL https://www.riigiteataja.ee/ akt/12771900._[Accessed Juny 29, 2013].

Šorohova, E., Kuuluvainen, T., Kangur, A., Jõgiste, K. 2009. Natural stand structures, disturbance regimes and successional dynamics in the Eurasian boreal forests: a review with special reference to Russian studies. - Annals of Forest Science, 66(2), $1-20$.

Simard, M., Lecomte, N., Bergeron, Y., Pare, D., Bernier P.Y. 2007. Forest productivity decline caused by successional paludification of boreal soils. - Ecological Applications, 16(7), 1619-1637.

Sustainability of State Forest Management 2010. Report of the National Audit Office to the Riigikogu. [WWW document]. - URL http:/ / www.riigikontroll. ee/tabid/206/Audit/2152/Area/15/language/enUS/Default.aspx [Accessed Juny 29, 2013].

Tyrrell, L.E., Crow, T.R. 1994. Structural Characteristics of Old-Growth Hemlock-Hardwood Forests in Relation to Age. - Ecology, 75(2), 370-386.

Winter, S. 2012. Forest naturalness assessment as a component of biodiversity monitoring and conservation management. - Forestry, 85(2), 293-304. 


\title{
Loodusdirektiivi metsaelupaikade puurinde koosseis, vanus ja jämeda puidu dünaamika Eestis 2010-2012. a. kogutud riiklike seireandmete põhjal
}

\author{
Anneli Palo ja Mark Gimbutas
}

\section{Kokkuvõte}

Euroopa Liidu loodusdirektiivi I Lisa elupaikade soodsa seisundi jälgimiseks peab iga liikmesriik neid seirama 6 aastase seiretsükliga, viimane aruanne esitati 2013. aastal. Pindalaliste muutuste kõrval näidatakse ka elupaikade struktuuri ja funktsioonide toimimist. Käesolevas artiklis analüüsitakse seiratud metsaelupaikade rinnete liigilist koosseisu, esimese rinde puude vanust ning jämeda puidu dünaamikat.

Loodusdirektiivi metsaelupaikades on puude keskmine vanus küll kõrgem Eestis keskmisest, kuid ületab napilt vastavate puuliikide raieküpsusvanust. Jämedate elavate puude keskmine diameeter on üldjuhul tunduvalt kõrgem jämedate surnud puude diameetrist, mis viitab metsade suktsessioonilisusele ja ajaloolisele majandatusele. Ulatuslikke suktsessioonilisi muutusi on oodata looduslikult arenevate puistute rinnete liigilises koosseisus. Leiti, et mittemajandatavates metsaelupaikades suureneb kuuse domineerivus, laialehiseid liike kohtab tulevikus tänasest sagedamini. Oluliselt väheneb aga männi, kase ja haava domineerimine esimese rinde koosseisus. Üldiselt metsaelupaikade rinnete koosseis mitmekesistub ehk liike on rohkem ja üksikliikide domineerivus väheneb. Loodusdirektiivi metsaelupaigatüüpide definitsioonide kohaselt tuleb osa muutunud peapuuliigiga metsaelupaiku ümber klassifitseerida. Mõnede elupaigatüüpide pindala suureneb ja elupaikade seisund paraneb tunduvalt, poollooduliku maakasutuse tulemusel tekkinud metsaelupaigatüüpide pindala aga väheneb ja struktuurilis-funktsionaalne kvaliteet mõnevõrra alaneb. Elupaigatüüpide seireandmete töötlemisel tuleb edaspidi eristada rangelt kaitstavate alade ja väljaspool neid asuvate alade metsaelupaiku. Rangelt kaitstavatel aladel on metsaelupaikade ümberklassifitseerimise põhjuseks looduslikud protsessid ja metsade seisund tervikuna paraneb. 